\title{
MULTI-RESOLUTION DECOMPOSITION APPLIED TO CRACKLE DETECTION
}

\author{
M. Du*\# F.K. Lam* F.H.Y. Chan* and J. Sun** \\ * Department of Electrical and Electronic Engineering, \\ The University of Hong Kong, Hong Kong. \\ ** Department of Electronic and Communication Engineering, \\ South China University of Technology, Guangzhou, China. 510641
}

\begin{abstract}
Crackles are one of the most important physical signs in clinical medicine. They have an explosive pattern in time domain, with a rapid onset and short duration. The timing, repeatability, and shape of crackles are important parameters for diagnosis. Therefore, automatic detection of crackles and their classification as fine and coarse crackles have important clinical value. Since the multiresolution decomposition technique can give high resolution in both time and frequency, it can be exploited to detect crackles and classify them according to the information in each scales. In this paper, we present a new method for crackle detection based on continuous wavelet transform. Theory, methods and experimental results are given in detail in this paper.
\end{abstract}

\section{INTRODUCTION}

Crackles are heard over the lungs in a variety of diseases. They convey diagnostic information. Crackles have an explosive pattern in time domain, with a rapid onset and short duration. The timings of inspiratory crackles are an important parameter of lung sound.[1] Crackle counts and their distribution over the chest wall have been used as objective signs of lung disease.

There are two kinds of crackles. Fine crackles are associated with conspicuous high frequency component while coarse crackles are associated with low frequency components. The classification of crackles as fine and coarse has a major clinical significance. The former are associated with interstitial lung diseases, while the latter are more prevalent in obstructive airway diseases.

Therefore, it is important to detect crackles and classify them as fine and coarse automatically.

An adaptive stationary-nonstationary nonlinear filter was used by Ono et al. to separate fine crackles from lung sound[2]. We also used predictive filter with adaptive threshold to detect crackles.[3] Murphy et al. classified crackles by measuring some parameters of the waveform in time domain.[4]. Munakata et al. developed criteria for separating fine and coarse crackles.[5]

In this paper, we use multi-resolution decomposition technique to detect and classify crackles automatically. We will show that this technique can give a good result for crackle detection and classification.

\section{CHARACTERISTICS OF CRACKLES}

Fine crackles which occur in patients with interstitial lung disease and early congestive heart failure, are heard during inspiration, and are high pitch. A typical fine crackle and its spectrum are shown in fig.1(a) and fig. 1(b) respectively.

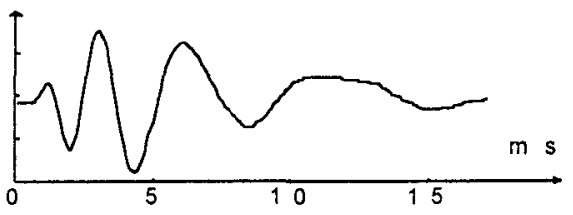

(a)

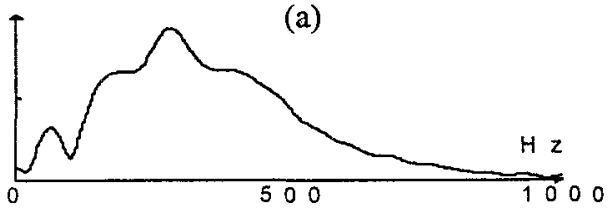

(b)

Fig. 1 A typical ine crackle.

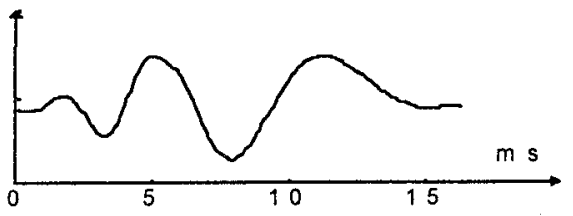

(a)

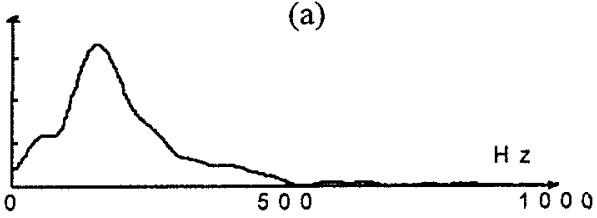

(b)

Fig 2. A typical coarse crackle

Coarse crackles which occur in patient with chronic bronchitis and severe pulmonary oedema, are heard during both inspiration and expiration, and are low pitch. A typical coarse crackle and its spectrum are shown in fig. 2. 
We can see from fig. 1 and fig.2 that the energy of the coarse crackle concentrates within about $160 \sim 220 \mathrm{~Hz}$ range while the fine crackle has much more high frequency components above $250 \mathrm{~Hz}$.

\section{WAVELET TRANSFORM AND MULTI- RESOLUTION DECOMPOSITION}

The continuous wavelet transform of a signal $x(t)$ is defined by:[6]

$c w t_{x}(\tau, s)=\frac{1}{s} \int x(t) \psi\left(\frac{t-\tau}{s}\right) d t$

where $\psi(t)$ is the mother wavelet and $\frac{1}{s} \psi\left(\frac{t-\tau}{s}\right)$ the wavelet basis function. The parameter $s$ is called the scale, and $\tau$ is the translation of the wavelet function along the time axis. The mother wavelet $\psi(t)$ must have the following properties: (1) $\int \psi(t) d t=0$, i.e., they have zero DC component; (2) it is a bandpass signal; (3) it decays rapidly towards zero with time. Examples of wavelet are Morlet, Shannon, Haar, and second derivation of Gaussian, etc..[6]

We can see from equation (1) that wavelet transform is no more than a computation of similarity between signal $x(t)$ and wavelet $\frac{1}{s} \psi\left(\frac{t-\tau}{s}\right)$. If we can design a wavelet which is similar to crackles, then, after wavelet transform is carried out, with noise suppressed, crackles can be located from a certain scale, and can also be classified as fine or coarse crackles according to the energy distribution at different scales.

\section{MULTIRESOLUTION DECOMPOSITION OF CRACKLES}

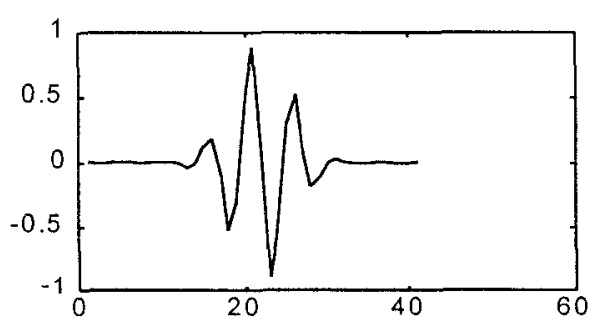

Figure 3. Wavelet in time domain

We use the wavelet, shown in fig. 3 , defined by:

$$
\psi(t)=\sin (12 \cdot t) \cdot e^{-t^{2} \cdot 4}
$$

This wavelet meets the admissible condition :

$$
C=\int_{-\infty}^{\infty} \frac{|\Psi(\omega)|^{2}}{|\omega|} d \omega<\infty
$$

and its shape is similar to crackles.

Fig.4(a) shows a raw lung sound signal. It contains two fine crackles. We use wavelet defined by (2) to perform the wavelet transform and get signals at each scale, shown in fig.4(b). The energy of signals at each scale can be obtained using the equation :

$$
E(s)=\int(c w t(\tau, s))^{2} d \tau
$$

and the result is shown in fig.4(c). It is obvious that it has a peak energy at scale 7 . This means that if we use a wavelet at scale 7 to perform wavelet transform, we can enhance fine crackles and suppress noise to a maximum extent.

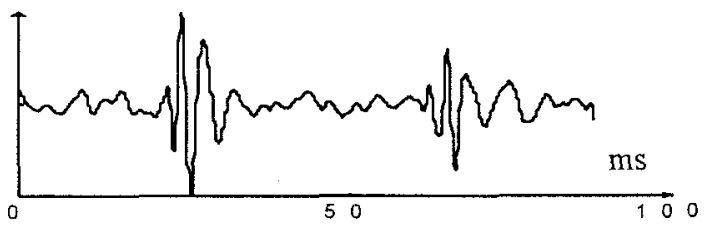

(a)

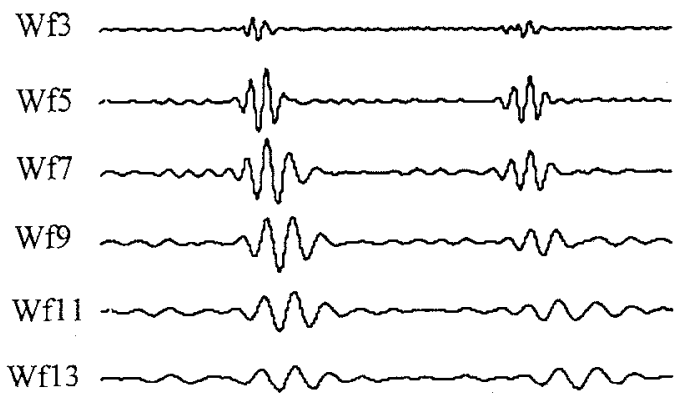

(b)

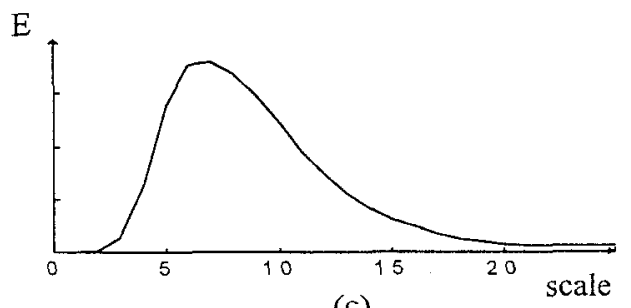

(c)

Figure 4. Multiresolution decomposition of a raw signal containing fine crackles.

Fig.5(a) shows a lung sound containing two coarse crackles. Following the processing procedure mentioned above, we can get the mutiresolution decomposition results, as shown in fig.5(b), and the 
energy distribution with scales, as shown in fig.5(c). It is clear that the peak energy of the decomposed signals is at scale 16.

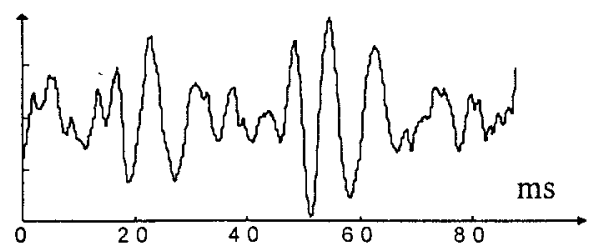

(a)

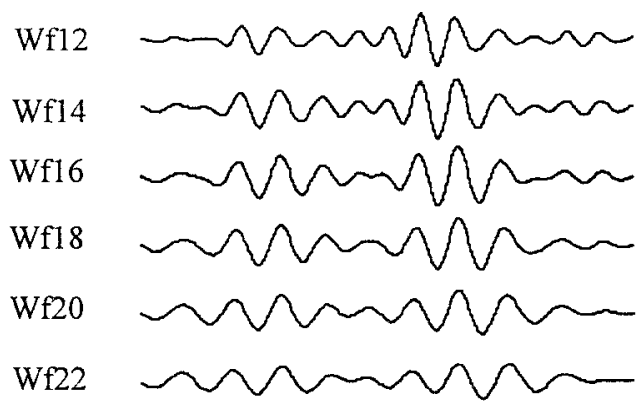

(b)

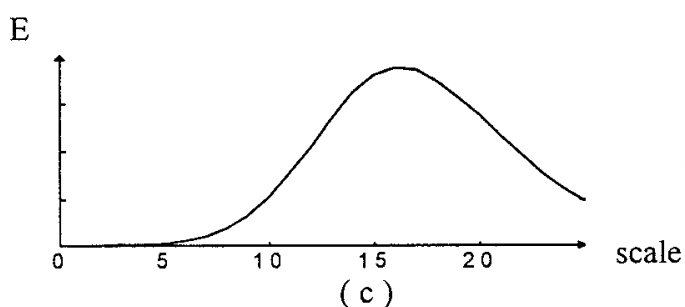

Fig.5 Multiresolution decomposition of a raw signal containing coarse crackles

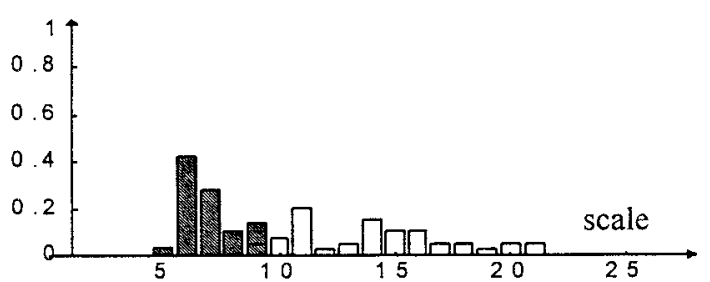

Fig.6 Statistical chart of peak energy distribution . Shadowed: fine crackles; Blank: coarse crackles

In order to obtain a criterion for separating fine and coarse crackles, we analyzed 50 fine crackles and 50 coarse crackles, and obtained the probability distribution of peak energy of decomposed signals for both fine and coarse crackles. The result is shown in fig.6.

From fig. 6 we can conclude that the optimum scale corresponding to peak energy among all scales for fine crackle decomposition are between 5 to 9 , while that for coarse crackles are between 10 to 21 . Therefore, we can easily classify crackles according the energy distribution in the multi-resolution decomposition results.

\section{NONLINEAR FILTER}

In order to detect crackle from the decomposed signal, we define a softhreshold which can adapt to the variation of signal energy. The threshold function is defined by :

$$
\operatorname{th}(s)=\sqrt{E(s)} \cdot e^{((s-2) / 10+1) / c 1} \cdot c 2
$$

where $c 1=4.0, c 2=1.0$ and $s$ is scale. $E(s)$ is the energy of decomposed signal at each scale, and can be computed using equation (3).

This threshold serves as a nonlinear filter and is applied to all decomposed signal to suppress noise further more.

\section{DETECTION AND CLASSIFICATION CRITERIA}

After filtering the decomposed signals, the envelope of each signal can be obtained. A peak of the envelope corresponds to the position of a crackle.

Energy of each filtered signal can be computed, and the energy distribution with scale can be obtained. If the peak energy lies between scale 5 to 8 , then the crackle can be regarded as a fine crackle. Otherwise, if the peak lies between scale 10 to 21 , the crackle can be classified as a coarse crackle. If the peak lies scale 9 , we can compare the mean energy on scale from 6 to 8 to that of scale 10 to 12 . If the former is greater than the latter, the crackle is classified as fine crackle; otherwise, it will be categorized into coarse crackle. This is because that fine crackles have more high frequency components and result in that the energy on the lower side (corresponding to high frequency) be greater than that of upper side. Coarse crackles have an opposite characteristic.

\section{CRACKLE DETECTION AND CLASSIFICATION EXPERIMENT}

Lung sounds are analyzed from a teaching tape by the American College of Chest Physicians. The signals are digitized using DT2831 A/D converter at a sampling rate of $10 \mathrm{kHz}$. A moving overlapped window is applied to the original signal to select a segment for analysis. Wavelet transform is applied to this segment of signal. The optimal scaic which has the maximum energy can be selected. According to the criteria discussed in section 4 , crackles can be detected and classified. The detection and classification block diagram is shown in fig. 7 . 


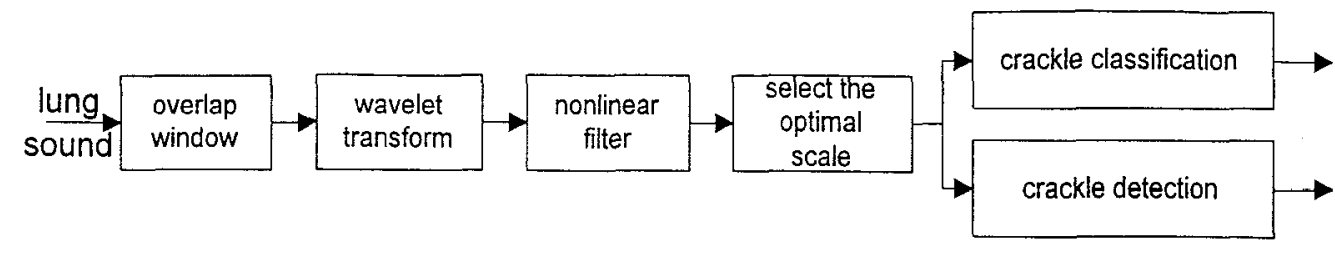

Fig.7 Block diagram for crackle detection and classification.

Fig8 and fig.9 show two experimental results. The original signals of these two figures are selected from the teaching tape defined as 'coarse' and 'fine' crackle respectively. We can see that not only large crackles can be detected correctly, but also weak crackles. We have compared the classification results with those from timeexpanded analysis proposed by Murphy[4], and confirmed that the classification was correct. Comparison was also made between this new method and traditional predictive filtering method for detection, and we found that this method could give a correct detection rate about $99.3 \%$ while the latter only $85.5 \%$.
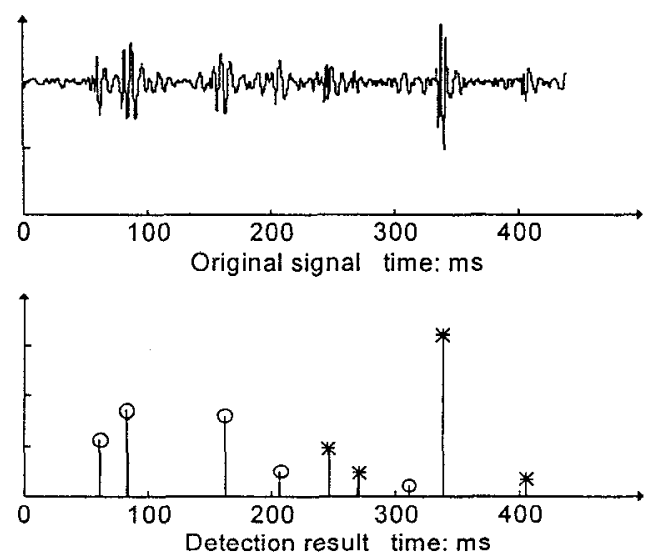

Fig. 8 Detection and classification results of a segment of 'coarse' crackle. * : fine; o : coarse
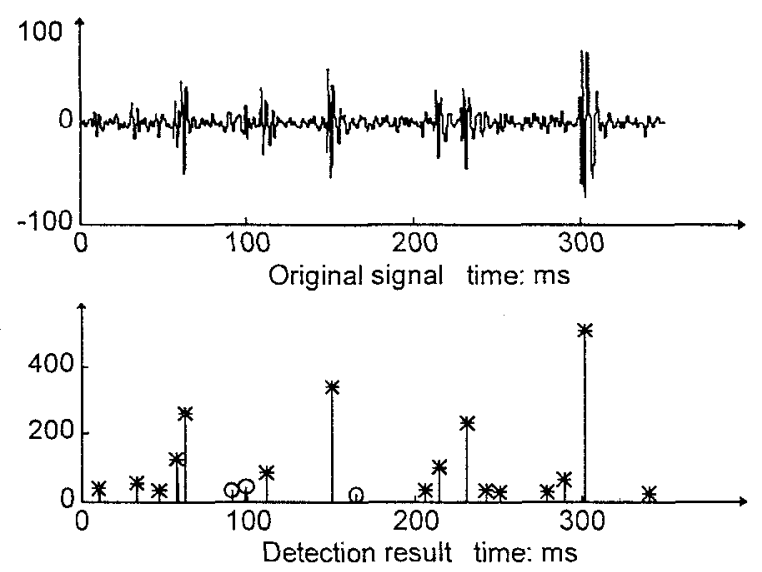

Fig.9 detection and classification results of a segment of 'fine' crackle. *: fine; o : coarse

\section{CONCLUSION}

Multiresolution decomposition technique has been used to detect and classify crackles. It is the first time to use a unified solution for both detection and classification of crackles. Comparing to the traditional predictive filtering for detecting crackles and time-expanded waveform analysis for classifying crackles, this technique can give a much better result. And it is easier to realize than the time-expanded method. Further works will be concentrated on modeling crackles and designing a matched wavelet.[9]

\section{REFERENCE}

[1] N. Gavrily and D. W. Cugell, Breath Sounds Methodology. CRC Press, Inc. . 1995

[2] M. Ono, K. Arakawa, M. Mori, et al. Separation of fine crackles from vesicular sounds by a nonlinear digital filter. IEEE Trans. Biomed. Eng. BME-36,286-291

[3] M. Du and J. Sun, Predictive filters applied to crackles detection. Proc. of China 13th Conference on Circuits and Systems. Chongqing, China, Oct. 1996. 152-155.

[4] R.L.H. Murphy, S.K. Holford, and W.C. Knowler, Visual lung-sound characterization by time-expanded waveform analysis. New Eng. J. Med., 296, 968-971, 1978.

[5] M. Munakata, H. Ukita, I. Doi, et al, Spectral and waveform characteristic of fine and coarse crackles. Thorax, 46, 1991. 651-657

[6] R, Y. Young, Wavelet theory and its applications, Kluwer Academic Publishers, 1993.

[7] S. Mallat, and W. L. Hwang, Singularity detection and processing with wavelet, IEEE Trans. on Information Theory. Vol.38, No.2 Mar 1992.

[8] J. Hoevers, and R.G. Loudon, Measuring crackles, Chest, 98,5, Nov. 1990. 1240-1243

[9] M. Du, F.H.Y. Chan, F.K. Lam, J. Sun, Crackle detection and classification based on matched wavelet analysis. submitted to 1997 IEEE Annual Conference of EBMS. Oct.1997, Chicago. USA.

"Visiting scholar from the Department of Electronic and Communication Engineering, South China University of Technology, Guangzhou, China. 